\title{
Differential metabolism of Mycoplasma species as revealed by their genomes
}

\author{
Fabricio B.M. Arraes ${ }^{1}$, Maria José A. de Carvalho $^{1}$, Andrea Q. Maranhão ${ }^{1}$, Marcelo M. Brígido ${ }^{1}$, \\ Fábio O. Pedrosa ${ }^{2}$ and Maria Sueli S. Felipe ${ }^{1}$ \\ ${ }^{1}$ Laboratório de Biologia Molecular, Departamento de Biologia Celular, Instituto de Ciências Biológicas, \\ Universidade de Brasília, Brasília, DF, Brazil. \\ ${ }^{2}$ Departamento de Bioquímica e Biologia Molecular, Universidade Federal do Paraná, Curitiba, PR, Brazil.
}

\begin{abstract}
The annotation and comparative analyses of the genomes of Mycoplasma synoviae and Mycoplasma hyopneumonie, as well as of other Mollicutes (a group of bacteria devoid of a rigid cell wall), has set the grounds for a global understanding of their metabolism and infection mechanisms. According to the annotation data, M. synoviae and $M$. hyopneumoniae are able to perform glycolytic metabolism, but do not possess the enzymatic machinery for citrate and glyoxylate cycles, gluconeogenesis and the pentose phosphate pathway. Both can synthesize ATP by lactic fermentation, but only M. synoviae can convert acetaldehyde to acetate. Also, our genome analysis revealed that $M$. synoviae and $M$. hyopneumoniae are not expected to synthesize polysaccharides, but they can take up a variety of carbohydrates via the phosphoenolpyruvate-dependent phosphotransferase system (PEP-PTS). Our data showed that these two organisms are unable to synthesize purine and pyrimidine de novo, since they only possess the sequences which encode salvage pathway enzymes. Comparative analyses of $M$. synoviae and $M$. hyopneumoniae with other Mollicutes have revealed differential genes in the former two genomes coding for enzymes that participate in carbohydrate, amino acid and nucleotide metabolism and host-pathogen interaction. The identification of these metabolic pathways will provide a better understanding of the biology and pathogenicity of these organisms.
\end{abstract}

Key words: Mollicutes, Mycoplasma, metabolism, comparative genomics.

Received: April 12, 2006; Accepted: October 9, 2006.

\section{Introduction}

Mollicutes (Latin "mollis" = soft, "cutis" = skin) are a group of bacteria devoid of a rigid cell wall. They are the smallest bacteria known $(0.2$ to $0.5 \mu \mathrm{m})$ and reportedly hold the smallest non-viral genomes known. The biology and pathogenicity of Mollicutes/Mycoplasma spp. have been extensively reviewed (Razin et al., 1998; Razin, 2005). Mollicutes are very fastidious in their nutritional requirements for in vitro growth and have limited biosynthetic capacity, a consequence of their concise genomes. Cholesterol is required for growth by species of Mycoplasma, Ureaplasma, Entomoplasma, Spiroplasma and Anaeroplasma. There is an apparent correlation between Mycoplasma growth rate and genome size: under optimal conditions, Mycoplasma pneumoniae M129 (genome size 816,394 bp) grows twice as fast as Mycoplasma

Send correspondence to Maria Sueli Soares Felipe. Laboratório de Biologia Molecular, Departamento de Biologia Celular, Instituto de Biociências, Universidade de Brasília, Campus Universitário, Asa Norte, 70910-900 Brasília, DF, Brazil. E-mail: msueli@unb.br. genitalium G-37 (genome size 580,074 bp) (Peterson and Fraser, 2001).

Ribosomal RNA (16S) phylogeny studies revealed that these bacteria evolved from cell-walled gram-positive Firmicutes of low $\mathrm{G}+\mathrm{C}$ content ( 23 to $40 \%$ ), probably of the genus Streptococcus [by shedding their genes to the probable minimum genome size in the range of $530 \mathrm{kbp}$ (Razin et al., 1998)], which appears to date to be the minimal gene assembly required for independent selfreplicating life, although not all of the genes included are deemed essential (Peterson and Fraser, 2001). Thus, the current hypothesis is that Mollicutes reductively evolved towards that minimal genome set, heavily dependent on the supply of nutrients from the host or a rich milieu. In this process, they have lost genes involved in biosynthesis of lipids, amino acids, cofactors and gram-positive-type cell wall components, as well as several genes involved in transcription regulation, cell division and heat shock response (Razin et al., 1998). Mycoplasma species are often deficient in intermediary energy metabolism, depending mostly on glycolysis as an ATP-generating system (Razin 
et al., 1998). This deficiency in metabolism, cellular processes and energy production imposes a lifestyle strictly dependent on the natural host. Therefore, Mollicutes are compulsory parasites/pathogens of humans (genera Mycoplasma and Ureaplasma), other vertebrates (Mycoplasma, Ureaplasma, Acholeplasma, Anaeroplasma and Asteroleplasma), insects (Entomoplasma, Mesoplasma, Spiroplasma and Phytoplasma) and plants (Entomoplasma, Mesoplasma, Spiroplasma, Acholeplasma and Phytoplasma) (Razin et al., 1998; Razin, 2005). The pathogenicity of poultry Mycoplasma has been recently reviewed (Bradbury, 2005).

The complete genome sequences of nine Mycoplasma species (including three different strains of $M$. hyopneumoniae) have been determined: M. gallisepticum R (genome size 996,422 bp), M. genitalium G-37 (580,074 bp), M. hyopneumoniae 232 (892,758 bp) 7448 (920,079 bp) and $\mathrm{J}(897,405 \mathrm{bp}), M$. mobile 163K (777,079 bp), $M$. mycoides subsp. mycoides SC strain PG1 (1,211,703 bp), M. penetrans HF-2 (1,358,633 bp), M. pneumoniae M129 (816,394 bp), M. pulmonis UAB CTIP (963,879 bp), and M. synoviae 53 (799,476 bp) (Entrez Genome). The complete genomes of one Ureaplasma species, Ureaplasma parvum (U. urealyticum) serovar 3 strain ATCC 700970 (751,719 bp) (Glass et al., 2000) and of Mesoplasma florum strain L1, (793,224 bp) are also known (Entrez Genome and references therein), as is that of the obligatory intracellular plant pathogen Phytoplasma (Candidatus Phytoplasma asteris, OY strain), with a genome size of about $860 \mathrm{kbp}$ (Oshima et al., 2004). No genome sequencing of species from the genera Entomoplasma, Spiroplasma, Acholeplasma, Anaeroplasma, and Asteroplasma was reported so far.

The concept of minimal genome has been extensively debated in both philosophical (Cho et al., 1999) and theoretical terms and tested experimentally (Peterson and Fraser, 2001); it was originally proposed by Morowitz (1984), who viewed Mycoplasma spp. as minimal organisms. In a comparative analysis of the genomes of $M$. genitalium and Haemophylus influenzae, Mushegian and Koonin (1996) concluded that 256 orthologous and non-orthologous genes sufficed to sustain the existence of a modern-type cell. The M. genitalium gene set of 480 is much larger then that, implying that it contains some non-essential genes, as probably other Mollicutes do too. Although Mollicutes carry the smallest genomes, they differ in gene complement size, which are probably a reflection of their efficient metabolic pathways and also a function of their host essential nutrient supply. As extensively discussed by Peterson and Fraser, 2001, the experimentally defined minimal genome is lineage-specific and, therefore, may not apply to different phylogenetic lineages (Peterson and Fraser, 2001; Zimmer, 2003; Razin et al., 1998).

In this paper we make a comparative analysis of the metabolic landscape of the genomes of seven Mycoplasma and one Ureaplasma species, highlighting mainly the extant differences among the differential sequences that encode enzymes that participate in carbohydrate, amino acid and nucleotide metabolism and host-pathogen interaction.

\section{Methods}

Mycoplasma sequences were obtained from the Brazilian Genome and Southern Genome Investigation Programs (both from LNCC) and from the National Center for Biotechnology Information (NCBI). Missing genes were mined with the TblastN program (Altschul et al., 1997) using known Mycoplasma proteins as query against $M$. synoviae and M. hyopneumoniae (strains J and 7448) genome databases. The Molligene database was also used to aid in comparative analysis. Metabolic pathways were defined using KEGG (Kyoto Encyclopedia of Genes and Genomes). In order to compare Mycoplasma enzyme coding sequences, we first found the missing EC numbers in $M$. synoviae and M. hyopneumoniae (strains J and 7448) (Table 1). An enzyme was considered absent only after their genome databases blast analysis. We also performed the same search in other Mycoplasma and Ureaplasma gene databases (Table 2).

\section{Results}

\section{General description of Mycoplasma synoviae and} Mycoplasma hyopneumoniae (strains J and 7448) metabolism

The genome project developed by the consortium of BRGene and Genesul reported the results of analyses of three complete Mycoplasma genomes - a pathogenic (7448) and a nonpathogenic (J) strain of the swine Mycoplasma hyopneumoniae and a strain of the avian pathogen Mycoplasma synoviae (Vasconcelos et al., 2005). By looking into aspects of Mycoplasma evolution, the authors found strain-specific regions, genome rearrangements and adhesion sequences in the $M$. hyopneumoniae strains, which are probably related to pathogenicity. Genome comparisons of those microorganisms revealed that its reduction was the consequence of a loss of metabolic pathways.

As concluded from genome data analysis, $M$. hyopneumoniae does not synthesize starch, glycogen, sucrose, pectin, sugar nucleotides such as UDP-glucose, ADP-glucose and others. These data also indicated that it can take up a variety of carbohydrates such as mono- and disaccharides (D-glucose, D-fructose, mannose, maltose, sucrose, inositol, sorbose, sorbitol, D-mannitol, etc.) via PEP-PTS (phosphoenolpyruvate-dependent phosphotransferase system). Only D-glucose and D-fructose are transformed into pyruvate by the glycolytic pathway. Pyruvate can be converted to lactate or acetyl-CoA. In the $M$. hyopneumoniae genome, we also found genes coding for enzymes that are able to convert both sugars to D-fructose6P and D-fructose-1,6-bisphosphate, which enter the 
Table 1 - Differential sequences encoding enzymes of Mycoplasma hyopneumoniae (strains J and 7448) and Mycoplasma synoviae.

\begin{tabular}{llccc}
\hline Enzyme Name & EC number & Mhy $(\mathrm{J})^{\mathrm{a}}$ & $M h y(7448)^{\mathrm{b}}$ & $M_{s y^{\mathrm{c}}}$ \\
\hline Mannitol - 1- phosphate - 5 - dehydrogenase & 1.1 .1 .17 & $\mathbf{X}$ & $\mathbf{X}$ & - \\
Myoinositol 2 - dehydrogenase & 1.1 .1 .18 & $\mathbf{X}$ & $\mathbf{X}$ & - \\
Dihydrofolate dehydrogenase & 1.5 .1 .3 & - & - & $\mathbf{X}$ \\
Fol D, methylenehydrofolate dehydrogenase (NADPH) & 1.5 .1 .5 & - & - & $\mathbf{X}$ \\
Pmsr; peptide methionine sulfoxide reductase & 1.8 .4 .5 & $\mathbf{X}$ & $\mathbf{X}$ & - \\
Thy A, thymidine synthase & 2.1 .1 .45 & - & - & $\mathbf{X}$ \\
Deoxyguanosine kinase & 2.7 .1 .113 & - & - & $\mathbf{X}$ \\
Glucokinase & 2.7 .1 .2 & $\mathbf{X}$ & $\mathbf{X}$ & - \\
Glycerol kinase & 2.7 .1 .30 & $\mathbf{X}$ & $\mathbf{X}$ & - \\
Pantetheine-phosphate adenylyltransferase & 2.7 .7 .3 & - & - & $\mathbf{X}$ \\
Holo-[acyl - carrier -protein] synthase & 2.7 .8 .7 & - & - & $\mathbf{X}$ \\
5'-nucleotidase & 3.1 .3 .5 & $\mathbf{X}$ & $\mathbf{X}$ & - \\
Phosphoprotein phosphatase & 3.1 .3 .16 & - & - & $\mathbf{X}$ \\
Pullulanase & 3.2 .1 .41 & $\mathbf{X}$ & $\mathbf{X}$ & - \\
Oligo-1, 6-glucosidase & 3.2 .1 .10 & - & - & $\mathbf{X}$ \\
Probable polypeptide deformylase & 3.5 .1 .88 & - & - & $\mathbf{X}$ \\
$N$-acetylneuraminate lyase & 4.1 .3 .3 & - & - & $\mathbf{X}$ \\
Citrate (pro-3S)-lyase & 4.1 .3 .6 & - & $\mathbf{X}$ & $\mathbf{X}$ \\
Mannose-6-phosphate isomerase & 5.3 .1 .8 & - & $\mathbf{X}$ \\
Phosphoglucomutase & 5.4 .2 .6 & & $\mathbf{X}$ \\
\hline
\end{tabular}

${ }^{\mathrm{a}}$ Mhy(J) - Mycoplasma hyopneumoniae J strain; ${ }^{\mathrm{b}}$ Mhy(7448) - Mycoplasma hyopneumoniae 7448 strain; ${ }^{\mathrm{c}}$ Msy(J) - Mycoplasma synoviae.

glycolytic pathway and can be used to produce dihydroxyacetone-P and glyceraldehyde-3P. M. hyopneumoniae also possesses genes coding for enzymes that phosphorylate lactose and galactitol to lactose-6P and galactitol-1P; convert glycerol to glyceraldehyde and glycerol-3P, and PEP into pyruvate and then to acetyl-CoA or lactate.

Similarly, M. synoviae data suggest that it does not synthesize starch, glycogen, sucrose, pectin, or sugar nucleotides such as UDP-glucose, ADP-glucose and others. It is probably able to take up extracellular substrates such as D-fructose, D-mannose, L-sorbose, D-sorbitol, D-mannitol and convert them to glyceraldehyde-3P, except mannose and mannitol, since these two substrates do not seem to be converted to fructose-6P. M. synoviae is probably unable to grow on either of these two sugars as sole carbon source.

M. hyopneumoniae has glucokinase but no hexokinase, and M. synoviae lacks the coding genes for both kinases. Both M. synoviae and M. hyopneumoniae could convert glycerol-3P into glyceraldehyde-3P with glycerone-P as an intermediate. Both organisms are unable to produce glucose-1P for starch synthesis, but can produce L-lactate and acetyl-CoA from pyruvate. M. synoviae can interconvert acetaldehyde and acetate by acetaldehyde dehydrogenase, but $M$. hyopneumoniae cannot. $M$. synoviae and M. hyopneumoniae are able to produce CoA from dephospho-CoA, but are unable to synthesize pantothenate and coenzyme A from pyruvate.
Both Mycoplasma spp. lack sequences encoding enzymes that participate in the citrate and glyoxylate cycles, gluconeogenesis (absence of fructose-1,6-bisphophatase), and the oxidative branch of the pentose phosphate pathway. They also lack all enzyme genes of the oxidative pathway, namely glucose-6P-dehydrogenase, 6P-gluconate-dehydrogenase and 6-phosphogluconate-lactonase. All genes coding for enzymes of the Entner-Doudoroff pathway seem to be absent in both organisms, so neither can convert D-glucose-6P into D-ribulose-5P.

Neither organism performs reactions of the glyoxylate cycle: the absence of isocitrate lyase and malate synthase coding sequences suggests that they are unable to synthesize glyoxylate and dicarboxylic acids such as oxaloacetate, succinate, citrate, isocitrate, cis-aconitate and malate. Both are devoid of all 1C-metabolism enzymes except for methylenetetrahydrofolate dehydrogenase in $M$. synoviae. The absence of an active glyoxylate cycle precludes growth of both $M$. synoviae and M. hyopneumoniae on 2-carbon substrates such as ethanol and acetate. In addition, both organisms are unable to perform gluconeogenesis, due to the absence of fructose-1,6-bisphosphatase and glucose-6-phosphatase genes.

It is important to mention that both organisms present genes involved in PRPP (phosphoribosyl-pyrophosphate) production. They are also capable to synthesize D-ribose$1 \mathrm{P}$ and 2-deoxy-D-ribose-1P for nucleoside generation. On 
Table 2 - Distribution of differential sequences encoding enzymes of other Mycoplasma and Ureaplasma species.

\begin{tabular}{|c|c|c|c|c|c|c|c|c|c|c|}
\hline Enzyme & EC\# & $M g a^{\mathrm{a}}$ & $M g e^{\mathrm{b}}$ & Mhy $232^{\mathrm{c}}$ & $M m o^{\mathrm{d}}$ & $M m y^{\mathrm{e}}$ & $M p e^{\mathrm{f}}$ & $M p n^{\mathrm{g}}$ & $M p u^{\mathrm{h}}$ & $U u^{\mathrm{i}}$ \\
\hline Mannitol - 1- phosphate - 5 - dehydrogenase & 1.1.1.17 & - & - & $\mathbf{X}$ & - & $\mathbf{X}$ & - & $\mathbf{X}$ & $\mathbf{X}$ & - \\
\hline Myoinositol 2 - dehydrogenase & 1.1.1.18 & - & - & $\mathbf{X}$ & - & - & - & - & - & - \\
\hline Dihydrofolate dehydrogenase & 1.5.1.3 & $\mathbf{X}$ & $\mathbf{X}$ & - & $\mathbf{X}$ & - & $\mathbf{X}$ & $\mathbf{X}$ & $\mathbf{X}$ & $\mathbf{X}$ \\
\hline Fol D, methylenehydrofolate dehydrogenase (NADPH) & 1.5.1.5 & $\mathbf{X}$ & $\mathbf{X}$ & - & $\mathbf{X}$ & $\mathbf{X}$ & $\mathbf{X}$ & $\mathbf{X}$ & $\mathbf{X}$ & $\mathbf{X}$ \\
\hline Pmsr; peptide methionine sulfoxide reductase & 1.8.4.5 & $\mathbf{X}$ & $\mathbf{X}$ & $\mathbf{X}$ & $\mathbf{X}$ & $\mathbf{X}$ & - & $\mathbf{X}$ & $\mathbf{X}$ & - \\
\hline Thy A, thymidine synthase & 2.1.1.45 & $\mathbf{X}$ & $\mathbf{X}$ & - & $\mathbf{X}$ & - & $\mathbf{X}$ & $\mathbf{X}$ & $\mathbf{X}$ & - \\
\hline Deoxyguanosine kinase & 2.7.1.113 & $\mathbf{X}$ & $\mathbf{X}$ & - & - & - & - & $\mathbf{X}$ & $\mathbf{X}$ & $\mathbf{X}$ \\
\hline Glucokinase & 2.7.1.2 & - & - & $\mathbf{X}$ & $\mathbf{X}$ & $\mathbf{X}$ & $\mathbf{X}$ & - & $\mathbf{X}$ & - \\
\hline Glycerol kinase & 2.7.1.30 & $\mathbf{X}$ & $\mathbf{X}$ & $\mathbf{X}$ & $\mathbf{X}$ & $\mathbf{X}$ & $\mathbf{X}$ & $\mathbf{X}$ & $\mathbf{X}$ & - \\
\hline Panthetheine-phosphate adenylyltransferase & 2.7.7.3 & $\mathbf{X}$ & - & - & $\mathbf{X}$ & $\mathbf{X}$ & $\mathbf{X}$ & - & $\mathbf{X}$ & \\
\hline Holo-[acyl - carrier -protein] synthase & 2.7.8.7 & $\mathbf{X}$ & $\mathbf{X}$ & - & $\mathbf{X}$ & $\mathbf{X}$ & $\mathbf{X}$ & $\mathbf{X}$ & $\mathbf{X}$ & $\mathbf{X}$ \\
\hline 5 '-nucleotidase & 3.1 .3 .5 & - & - & $\mathbf{X}$ & $\mathbf{X}$ & - & - & - & $\mathbf{X}$ & - \\
\hline Phosphoprotein phosphatase & 3.1.3.16 & $\mathbf{X}$ & $\mathbf{X}$ & - & $\mathbf{X}$ & $\mathbf{X}$ & $\mathbf{X}$ & $\mathbf{X}$ & $\mathbf{X}$ & $\mathbf{X}$ \\
\hline Pullulanase & 3.2.1.41 & - & - & $\mathbf{X}$ & $\mathbf{X}$ & - & - & - & $\mathbf{X}$ & - \\
\hline Oligo-1, 6-glucosidase & 3.2 .1 .10 & - & - & - & $\mathbf{X}$ & $\mathbf{X}$ & - & - & $\mathbf{X}$ & - \\
\hline Probable polypeptide deformylase & 3.5.1.88 & $\mathbf{X}$ & $\mathbf{X}$ & - & $\mathbf{X}$ & $\mathbf{X}$ & $\mathbf{X}$ & $\mathbf{X}$ & $\mathbf{X}$ & $\mathbf{X}$ \\
\hline $\mathrm{N}$-acetylneuraminate lyase & 4.1.3.3 & - & - & - & - & $\mathbf{X}$ & - & - & - & - \\
\hline Citrate (pro-3S)-lyase & 4.1.3.6 & $\mathbf{X}$ & - & - & - & - & - & - & - & - \\
\hline Mannose-6-phosphate isomerase & 5.3 .1 .8 & $\mathbf{X}$ & - & $\mathbf{X}$ & $\mathbf{X}$ & $\mathbf{X}$ & $\mathbf{X}$ & - & $\mathbf{X}$ & - \\
\hline Phosphoglucomutase & 5.4.2.6 & $\mathbf{X}$ & - & - & $\mathbf{X}$ & - & - & - & $\mathbf{X}$ & - \\
\hline
\end{tabular}

${ }^{\mathrm{a}}$ Mga - Mycoplasma galysepticum; ${ }^{\mathrm{b}}$ Mge - Mycoplasma genitalium; ${ }^{\mathrm{c}}$ Mhy (232)- Mycoplasma hyopneumoniae strain $232 ;{ }^{\mathrm{d}}$ Mmo - Mycoplasma mobile $163 \mathrm{~K} ;{ }^{\mathrm{e}}$ Mmy - Mycoplasma mycoides; ${ }^{\mathrm{f}}$ Mpe - Mycoplasma penetrans HF2; ${ }^{\mathrm{g}}$ Mpn - Mycoplasma pneumoniae M129; ${ }^{\mathrm{h}}$ Mpu - Mycoplasma pulmonis UAB CTIP; ${ }^{\mathrm{i}} U$ u - Ureaplasma urealyticus.

the other hand, they are unable to synthesize purines and pyrimidines de novo. They only present salvage pathwaysencoding sequences for nucleotide biosynthesis.

M. hyopneumoniae lacks the genes coding for fatty acid biosynthesis and degradation pathway enzymes, as well as those for sterol, bile acid, carotenoid and vitamins K and E biosynthesis. Ketone bodies, C21 steroid hormone, androgen and estrogen metabolic pathways are also absent. However, M. synoviae and M. hyopneumoniae can produce cardiolipin and ceramide, the only two membrane components identified so far. Both $M$. synoviae and $M$. hyopneumoniae can synthesize cardiolipin from acylglycerol-3P through a pathway involving diacylglycerol-3-P and CDP-diacylglycerol. Cardiolipin is found only in membranes of bacteria and mitochondria, i.e., organisms/ organelles whose function is to generate an electrochemical potential for substrate transport and ATP synthesis. Neither organism studied synthesizes triacylglycerol, lecithin, glycolipids or plasmalogens. Cardiolipin is apparently the only membrane lipid of Mycoplasma spp., and both lack all kinds of lipase genes.

Our data suggest that $M$. hyopneumoniae synthesizes no amino acid at all. On the other hand, both the $M$. synoviae and the $M$. hyopneumoniae genomes have all the enzyme-coding sequences necessary for the synthesis of all aminoacyl-tRNAs but glutaminyl-tRNA from glutamine.
The glutamine-tRNA ligase or glutaminyl-tRNA synthetase is absent in both organisms, but they have the amido ligase to convert glutamyl-tRNA into glutaminyltRNA. The gene for the methionyl-tRNA formyltransferase enzyme is present in $M$. synoviae, but absent in $M$. hyopneumoniae. Apparently, in M. hyopneumoniae the initiator tRNA for protein synthesis is L-methionyl-tRNA. Neither M. synoviae nor M. hyopneumoniae synthesizes either alanine or aspartate from pyruvate or oxaloacetate. They have alanyl-tRNA synthetase, aspartyl and asparaginyl-tRNA synthetase-coding genes, but lack all others for the corresponding pathways. Both organisms are unable to synthesize glutamate or glutamine, but are able to interconvert serine and glycine via glycine hydroxymethyltransferase. Also, they present glycine, serine and threonine-tRNA ligases and are able to perform the oxi-reduction reactions of lipoylproteins. Both are unable to synthesize glycine, serine or threonine, which must be taken up from the environment, which probably accounts for their known auxotrophic behavior regarding these amino acids. Both seem to produce glycine and trimethylammonium butanol from N-6-hydroxy-trimethyl-lysine. Only M. synoviae is capable to produce 4-tri-methylammonium butanoate from tri-methylammonium butanol. Both organisms lack all other lysine degradation enzyme genes. They also produce S-adenosyl methionine from 
L-methionine. M. hyopneumoniae oxidizes L-methionine to L-methionine sulfoxide, while $M$. synoviae presents asparagine synthetase. Neither is able to synthesize or degrade phenylalanine.

Neither M. synoviae nor M. hyopneumoniae presents any coding sequence for enzymes of the nitrogen cycle. The nitrification, denitrification, nitrate and ammonium assimilation pathways are absent. Both are unable to synthesize nitrogenous compounds, including ammonium (even from $\mathrm{N}$-compounds) and amino acids. The only exception is the potential ability of $M$. synoviae to synthesize asparagine from aspartate and ammonia.

Genes encoding enzymes for the respiratory chain are not found in M. synoviae or M. hyopneumoniae (strains J and 7448). Sequences related to ATP synthases subunits are not detected, except for ADP tetraphosphate phosphohydrolase. Both M. synoviae and M. hyopneumoniae lack all the cytochrome complement and therefore cannot perform electron transport or oxidative phosphorylation. They apparently produce ATP mainly through the glycolytic pathway.

Both bacteria are unable to synthesize chitin, glycosaminoglycans, lipopolysaccharides, peptideoglycans, mucopolysaccharides or lipoglycoproteins, as they do not have genes coding for enzymes that synthesize either UDP-Nacetyl D-glucosamine or UDP-N-acetyl D-mannosamine.

Remarkable is the potential of both Mycoplasmas to produce aerobactin from N-6-acetyl-N-6-hydroxylysine. Aerobactin is a siderophore involved in iron uptake and virulence in bacteria. Both $M$. synoviae and $M$. hyopneumoniae present the coding sequences that make them able to produce the ceramide ciliatine from CMPciliatine and 2-hydroxypropyl-phosphonate from phosphonoacetaldehyde.

\section{Comparison of some Mycoplasma species and Ureaplasma urealyticus genomes: differential enzyme coding sequences}

The differential enzymes for carbohydrate metabolism of Mollicutes analyzed here are: glucokinase (EC 2.7.1.2), phosphoglucomutase (EC 5.4.2.6), mannose-6phosphate-isomerase (EC 5.3.1.8), ATP citrate (pro-3S) lyase (EC 4.1.3.6), pullulanase (EC 3.2.1.41), and oligo1,6-glucosidase (EC 3.2.1.10). Glucokinase, an essential enzyme for the entry of D-glucose in the glycolytic pathway, was found in all strains of $M$. hyopneumoniae, $M$. pulmonis, M. mobile, M. mycoides, M. penetrans, $M$. pneumoniae and U. urealyticus. These Mollicutes should be able to use glucose as a carbon source for this metabolic pathway. An alternative form of activating glucose for the glycolysis pathway is through the activity of phosphoglucomutase that catalyzes the reversible conversion of glucose-1-phosphate to glucose-6-phosphate. The gene that encodes this enzyme was found in some Mollicutes, namely $M$. synoviae, $M$. mobile, $M$. pulmonis and $M$. galysepticum, but not in M. hyopneumoniae (all analyzed strains). Also, in these organisms the enzyme mannose6-phosphate isomerase catalyzes the interconversion of fructose-6-phosphate (which can be also used in the glycolytic pathway) and mannose-6-phosphate, which could also be catabolized through a mannose pathway (Pitkänen et al., 2004). As shown in Table 2, the gene that encodes this enzyme was absent only in $M$. synoviae, $M$. pneumoniae and M. galysepticum, which may mean that these organisms need diversified carbon sources for survival in their respective hosts. The ATP citrate (pro-3S) lyase is an enzyme that breaks down citrate into acetate and oxaloacetate (Hoffmann et al., 1979). Analyzing the data, M. synoviae is the only one amongst all Mollicutes analyzed in this study that presents the gene encoding this enzyme. For these intracellular pathogens, there must be the alternative of citrate breakdown by the host, so they can use oxaloacetate and acetate for ATP synthesis. Glucose is found in the cell mainly as starch and/or glycogen polysaccharides. Although the Mollicutes are simple organisms, some of them, like M. hyopneumoniae (all analyzed strains), M. mobile and M. pulmonis, have in their genome the gene that encodes the enzyme pullulanase that hydrolyses $(1,6)-\alpha$-D-glucosidic linkages in polysaccharides such as amylopectin and glycogen, converting them into glucose. This feature can be important in the host-pathogen interaction. Pullulanase degrades glycogen to maltose, which can be utilized by the enzyme oligo-1,6-glucosidase that completes degradation to glucose (Doman-Pytka and Bardowski, 2004). M. synoviae is thought to produce organic acids from maltose, which is further supported by the finding of sequences encoding the enzyme oligo-1,6-glucosidase, which is actually detected only in M. synoviae and in M. pulmonis.

The gene encoding the enzyme myoinositol2-dehydrogenase (EC 1.1.1.18) was identified only in $M$. hyopneumoniae (all analyzed strains). This enzyme catalyzes myoinositol conversion to glyceraldehyde-3P, which enters in the glycolytic pathway as an intermediate. As $M$. hyopneumoniae has only this enzyme of a pathway that comprises five others, this specific dehydrogenase should act on another substrate or participate in another cellular event such as oxidative stress. Eze et al. (1987) showed that superoxide dismutase or parabenzoquinone inhibit the myoinositol-2-dehydrogenase of Bacillus pumilus. They discovered the involvement of this enzyme in the transference of electrons and the production of the catalytic superoxide intermediate $\left(\mathrm{O}^{2-}\right)$ and/or the free radical hydroxyl $(\mathrm{OH})$, which can be important for host-pathogen interactions in Mollicutes. A successful pathogen must be able to overcome or suppress this complex array of host defenses mediated by reactive oxygen species (ROS). In fact, microbial suppression of ROS-mediated defenses by secretion of ROS-scavenging enzymes such as superoxide dismutase and catalase, which convert ROS into less reactive species, 
has been extensively documented in both plant and animal pathogens (San Mateo et al., 1998). Evidence is also emerging that pathogens suppress ROS-mediated defenses by non-enzymatic quenching of ROS. Mannitol has long been recognized as a potent ROS quencher in vitro, and has widely been used as a laboratory reagent to scavenge hydroxyl radicals generated by the phagocyte respiratory burst or by cell-free oxidant systems (Tauber and Babior, 1977). In vivo, increased mannitol production protects Saccharomyces cerevisiae from oxidative injury (Chaturvedi et al., 1997). Furthermore, it was recently shown that the human fungal pathogen Cryptococcus neoformans produces mannitol to quench neutrophil-generated ROS and thereby suppress this animal defense (Chaturvedi et al., 1996). The presence of the gene that encodes mannitol1-phosphate-5-dehydrogenase (EC 1.1.1.17) in $M$. hyopneumoniae (all analyzed strains), M. mycoides, $M$. pneumoniae and M. pulmonis is important to the conversion of D-mannitol-1-phosphate into D-fructose-6-phosphate, by the reduction of $\mathrm{NAD}^{+}$to NADH. This reaction removes the reactive oxygen species of the cell by mannitol degradation; supplies the glycolytic pathway with Dfructose-6-phosphate, and also assists in the maintenance of the cell redox balance by means of NADH production. Amino acids vary in susceptibility to oxidative damage, with methionine residues as the most vulnerable, followed by cysteine and tyrosine (Levine et al., 1996). ROS readily oxidizes methionine residues by two electrons to form sulfoxide, which, in turn, can be reduced back to methionine by the peptide methionine sulfoxide reductase (Pmsr EC 1.8.4.5). According to sequencing data, the gene that encodes this enzyme was found in all Mollicutes except in $M$. synoviae, $M$. penetrans and $U$. urealyticus, which is consistent with its importance in the oxidative damage response.

All nascent polypeptides in bacteria, chloroplasts and mitochondria contain an $N$-terminal formyl-methionine (Meinnel et al., 1993). In bacteria, peptide deformylase (PDF - EC 3.5.1.88) removes the formyl group from the majority of nascent polypeptides (Pei, 2001). Upon bacterial infection or tissue damage, these $N$-formylated peptides are released into the affected area, triggering a cascade of immunologic responses and the migration of leukocytes to the site of infection or injury. Of all Mollicutes analyzed in this work, the gene encoding the peptide deformylase was absent only in M. hyopneumoniae. This fact may imply a differential mechanism of this Mollicute to escape the host immune system.

Sialic acids are a large family of nine-carbon polyhydroxylated $\alpha$-ketoacids that play a wide variety of roles in nature (Angata and Varki, 2002). Most of them are derivatives of the common core structures $N$-acetylneuraminate acid or 2-keto-3-deoxy-D-glycero-D-galacto-nonulosonic acid. As a result of their extracellular localization, the sialic acids play important roles in mediating cellular recogni- tion, adhesion processes (Varki, 1997) and recognition by the lectins of the inflammatory and immune response pathway mechanisms (Vestweber and Blanks, 1999). The presence of the gene that encodes the enzyme $N$-acetylneuraminate lyase (EC 4.1.3.3) in M. synoviae is an evidence of a potentially relevant mechanism of infection. $\mathrm{N}$-acetylneuraminate lyase degrades $\mathrm{N}$-acetylneuraminate acid to $\mathrm{N}$ acetyl-D-mannosamine and pyruvate, which can be used in ATP synthesis. Moreover, this enzyme can be an evolutionary remnant, since it is involved in peptidoglycan metabolism in bacteria, which is absent in Mycoplasma spp.

Mycoplasma species are unable to synthesize purine and pyrimidine by de novo pathways. Therefore, salvage of pre-existing nucleosides and bases is essential for their survival; they depend on pathways that use either nucleobases or nucleosides to synthesize the respective nucleoside triphosphates/deoxynucleoside triphosphates. Two paralogs of deoxyguanosine kinase (EC 2.7.1.113) are found in $M$. synoviae, M. pulmonis, M. pneumoniae and $U$. urealyticus. This enzyme transfers a phosphate from ATP to deoxyguanosine, forming deoxyguanosine monophosphate (dGMP). M. hyopneumoniae has an alternative deoxiguanosine kinase activity, supplied by a 5' ribonucleotide phosphohydrolase enzyme (EC 3.1.3.5), also found in M. pulmonis. This ribonuclease is essential for Corynebacterium glutamicum, which secretes it to acquire inorganic phosphate by using nucleotides as phosphorus source (Rittmann et al., 2005).

M. hyopneumoniae cannot synthese tetrahydrofolate from folate, since it lacks the enzymes dihydrofolate dehydrogenase (EC 1.5.1.3) and methylenehydrofolate dehydrogenase (Fol D - EC 1.5.1.5). Moreover, the absence of thymidine synthase (EC 2.1.1.45), also absent in $U$. urealyticum, accounts for a presumable dependence of $M$. hyopneumoniae on this cofactor, that must be obtained from the host.

Approximately $4 \%-5 \%$ of all reactions in the intermediary metabolism rely on coenzyme $\mathrm{A}$ as an essential cofactor (Begley et al., 2001). So, the presence of enzymes like pantetheine-phosphate adenylyltransferase (EC 2.7.7.3) and holo-[acyl carrier protein] synthase (EC 2.7.8.7), which work together in coenzyme A biosynthesis (Morris and Izard, 2004), is essential for the metabolism of this cofactor. Pantetheine-phosphate adenylyltransferase was found only in M. synoviae, M. mobile, M. mycoides, M. penetrans and $M$. pulmonis and not in the other Mollicutes, whereas the holo-[acyl carrier protein] synthase was found in all Mollicutes except $M$. hyopneumoniae (all analyzed strains).

\section{Concluding Remarks}

The bioinformatics analysis of the M. synoviae and M. hyopneumoniae (strains J and 7448) genome database has made the description of their global metabolism possible. Also, comparative analyses of similar data from other 
Mollicutes enabled the identification of the main metabolic points of divergence. The identification of metabolic pathways is likely to provide a better understanding of these organisms, as well as to help finding more effective treatments for the respective diseases. M. synoviae and $M$. hyopneumoniae have a simple genome and are able to perform only the most essential metabolic processes of energy production, survival and host-pathogen interaction. The data presented here may aid in experimental design to investigate Mycoplasma metabolism further. Taken all results together, M. synoviae and M. hyopneumoniae can synthesize ATP via the glycolytic pathway or lactic fermentation, but these two pathogens are unable to perform the citrate and glyoxylate cycles, respiratory chain, gluconeogenesis, and pentose phosphate pathway reactions. $M$. hyopneumoniae does not have the pathways for fatty acid biosynthesis and degradation, but $M$. synoviae and $M$. hyopneumoniae can produce cardiolipin and ceramide, the only two membrane components identified in the genus. Nucleotide biosynthesis is performed in these two pathogens only by salvage pathways and they have enzymes for the synthesis of all aminoacyl-tRNAs, although $M$. hyopneumoniae synthesizes no amino acid at all. Finally, since our results were obtained by in silico study, experimental validation of these data will be needed.

\section{Acknowledgments}

The authors are dearly indebted to Hugo Costa Paes for text revision.

\section{References}

Altschul SF, Madden TL, Schaffer AA, Zhang J, Zhang Z, Miller W and Lipman DJ (1997) Gapped BLAST and PSI-BLAST: A new generation of protein database search programs. Nucleic Acids Res 25:3389-33402.

Angata T and Varki A (2002) Chemical diversity in the sialic acids and related alpha-keto acids: An evolutionary perspective. Chem Rev 102:439-469.

Begley TP, Kinsland C and Strauss E (2001) The biosynthesis of coenzyme A in bacteria. Vitam Horm 61:157-171.

Bradbury JM (2005) Poultry mycoplasmas: Sophisticated pathogens in simple guise. Br Poult Sci 46:125-136 (in: Gordon Memorial Lecture).

Chaturvedi V, Bartiss A and Wong B (1997) Expression of bacterial mtlD in Saccharomyces cerevisiae results in mannitol synthesis and protects a glycerol-defective mutant from high-salt and oxidative stress. J Bacteriol 179:157-162.

Chaturvedi V, Wong B and Newman SL (1996) Oxidative killing of Cryptococcus neoformans by human neutrophils. Evidence that fungal mannitol protects by scavenging reactive oxygen intermediates. J Immunol 1156:3836-3840.

Cho MK, Magnus D, Caplan AL, McGee D and the Ethics of Genomics Group (1999) Ethical considerations in synthesizing a minimal genome. Science 286:2087-2090.

Doman-Pytka M and Bardowski J (2004) Pullulan degrading enzymes of bacterial origin. Crit Rev Microbiol 30:107-121.
Eze MO, Nnamani LC, Ojiako RI and Ogan AU (1987) Effects of oxygen free radical scavengers on the membrane myoinositol dehydrogenase of Bacillus pumilus strain 5. Free Radic Res Commun 4:105-108.

Glass JI, Lefkowitz EJ, Glass JS, Heiner CR, Chen EY and Cassell $\mathrm{GH}$ (2000) The complete sequence of the mucosal pathogen Ureaplasma urealyticum. Nature 407:757-762.

Hoffmann GE, Kreisel C, Wieland OH and Weiss L (1979) Pyruvate dehydrogenase and ATP citrate (pro-3S)-lyase activities in adipose tissue and liver of the young lean and the older obese rat. Hoppe Seylers Z Physiol Chem 360:45-50.

Levine RL, Mosoni L, Berlett BS and Stadtman ER (1996) Methionine residues as endogenous antioxidants in proteins. Proc Natl Acad Sci USA 93:15036-15040.

Meinnel T, Mechulam Y and Blanquet S (1993) Methionine as translation start signal: A review of the enzymes of the pathway in Escherichia coli. Biochimie 75:1061-1075.

Morowitz HJ (1984) The completeness of molecular biology. Isr J Med Sci 20:750-753.

Morris VK and Izard T (2004) For the record: Substrate-induced asymmetry and channel closure revealed by the apoenzyme structure of Mycobacterium tuberculosis phosphopantetheine adenylyltransferase. Protein Sci 13:2547-2552.

Mushegian AR and Koonin EV (1996) A minimal gene set for cellular life derived by comparison of complete bacterial genomes. Proc Natl Acad Sci USA 93:10268-10273.

Oshima K, Kakizawa S, Nishigawa H, Jung HY, Wei W, Suzuki S, Arashida R, Nakata D, Miyata S, Ugaki M and Namba S (2004) Reductive evolution suggested from the complete genome sequence of a plant-pathogenic phytoplasma. Nat Genet 36:27-29.

Pei D (2001) Peptide deformylase: A target for novel antibiotics? Expert Opin Ther Targets 5:23-40.

Peterson SN and Fraser CM (2001) The complexity of simplicity Genome Biol 2: Comment2002.1-2002.8. http:// genomebiology.com/2001/2/2/comment/2002.

Pitkänen JP, Törma A, Alff S, Huopaniemi L, Mattila P and Renkonen R (2004) Excess mannose limits the growth of phosphomannose isomerase PMI40 deletion strain of Saccharomyces cerevisiae. J Biol Chem 279:55737-55743.

Razin S (2005) The genus Mycoplasma and related genera (class Mollicutes). In: Dworkin M et al (eds) The Prokaryotes: An Evolving Electronic Resource for the Microbiological Community. 3rd ed. release 3.19. Springer-Verlag, New York, http://link.springer-ny.com/link/service/books/10125/.

Razin S, Yogev D and Naot Y (1998) Molecular biology and pathogenicity of mycoplasmas. Microbiol Mol Biol Rev 62:1094-1156.

Rittmann D, Sorger-Herrmann U and Wendisch VF (2005) Phosphate starvation-inducible gene ushA encodes a 5' nucleotidase required for growth of Corynebacterium glutamicum on media with nucleotides as the phosphorus source. Appl Environ Microbiol 71:4339-4344.

San Mateo LR, Hobbs MM and Kawula TH (1998) Periplasmic copper-zinc superoxide dismutase protects Haemophilus ducreyi from exogenous superoxide. Mol Microbiol 27:391-404.

Tauber AI and Babior BM (1977) Evidence for hydroxyl radical production by human neutrophils. J Clin Invest 60:374379. 
Varki A (1997) Sialic acids as ligands in recognition phenomena. FASEB J 11:248-255

Vasconcelos AT, Ferreira HB, Bizarro CV, Bonatto SL, Carvalho MO, Pinto PM, Almeida DF, Almeida LG, Almeida R, Alves-Filho L, et al. (2005) Swine and poultry pathogens: The complete genome sequences of two strains of Mycoplasma hyopneumoniae and a strain of Mycoplasma synoviae. J Bacteriol 187:5568-5577.

Vestweber D and Blanks JE (1999) Mechanisms that regulate the function of the selectins and their ligands. Physiol Rev 79:181-213.

Zimmer C (2003) Tinker, tailor: Can Venter stitch together a genome from scratch? Science 299:1006-1007.

\section{Internet Resources}

Brazilian Genome. http://www.brgene.lncc.br. (October, 2005)]. Entrez Genome. http://www.ncbi.nlm.nih.gov/genomes/static/ eub_g.html (September, 2005).

Kyoto Encyclopedia of Genes and Genomes (KEGG), http:// www.genome.jp/kegg/ (October, 2005).

Molligene database, http://cbi.labri.fr/outils/molligen (October, 2005).

National Center for Biotechnology Information (NCBI), http:// www.ncbi.nlm.nih.gov. (October, 2005).

Southern Genome Investigation Program, http://www.genesul. lncc.br. (October, 2005).

Associate Editor: Arnaldo Zaha 